\title{
Survey of Agent-Based Simulations for Modelling COVID-19 Pandemic
}

\author{
Abdulla M. Alsharhan* \\ Faculty of Engineering \&IT, The British University in Dubai, 345015, UAE
}

\begin{tabular}{l} 
A R T I C L E I N F O \\
\hline Article history: \\
Received: 11 December, 2020 \\
Accepted: 08 March, 2021 \\
Online: 17 March, 2021 \\
\hline
\end{tabular}

Keywords:

COVID-19

Agent-Based Models

Agent-Based Simulations,

Pandemic simulation

Individual-Based Model

\begin{abstract}
A B S T R A C T
On the 11th of March 2020, the World Health Organization (WHO) declared COVID-19 as a pandemic. Part of controlling measures of the pandemic is to understand the disease's trajectories. There are several possible interventions that can prevent and control its spread. Determining an optimal strategy is critical for policymakers to understand the impact of different scenarios. Many modelers are using agent-based simulations to virtually examine the efficiency of these scenarios. This paper aims to review published papers that discussed Agent-based simulation (ABS) for modelling the COVID-19 pandemic. Major databases were searched for published articles in 2020 from top-ranking journals. Ten published papers were carefully chosen, and their findings were summarized and discussed. Among the methods used, three ABS models were shared as open source. Major findings included mask-wearing and working/studying from home as the optimal strategies, whereas airport screening is insufficient, and vertical isolation is similar to 'doing nothing' scenarios. Finally, one paper discussed the gaps in ABS and proposed a call of actions to the scientific community and guidelines to responsibly improve the ABS modelling's quality. This paper can contribute to understanding the current landscape of the COVID19 pandemic simulation models and their limitations. It is proposed to access selected opensourced agent-based models to evaluate, utilize, customize or learn from, to help conduct more accurate simulations.
\end{abstract}

\section{Introduction}

The city of Wuhan witnessed a viral outbreak for the first time in December 2019. Since then, the virus was classified as a zoonotic coronavirus, belonging to the same family of the Severe Acute Respiratory Syndrome (SARS) coronavirus and the Middle East Respiratory Syndrome (MERS) coronavirus. The novel virus was later named SARS-CoV-2. By February 2020, 33,738 infections were reported in China with 811 deaths. On the $30^{\text {th }}$ of January 2020, The World Health Organization (WHO) confirmed the outbreak as a Public Health Emergency of International Concern. On the $11^{\text {th }}$ of March, the same organization declared COVID-19 as a pandemic [1].

COVID-19 has brought many challenges to humanity, including a demand on novel medical treatments, social policies and economic initiatives. The fast response from the scientific community included dedicating studies from two perspectives to deal with the pandemic: researches focusing on treatment and diagnostic tools, and another dealing with the viral spread

${ }^{*}$ Corresponding Author: Abdulla M. Alsharhan, UAE, +971568284998, alsharhan@outlook.com

www.astesj.com

https://dx.doi.org/10.25046/aj060250 forecasting models [2]. While tight social distancing measures have proven effective in slowing down the viral spread; it was expected that relaxing some of these constraints will result in a second wave of spread [3]. Computational models provide quantitative support to public health teams and policy makers' in their readiness [4]. One of the most useful insights provided by computational model simulations is its ability to virtually explain natural phenomena, which are either not possible to simulate in a real-world, or too costly to be simulated. These simulations can support decision-makers in studying the current plans' efficiency and assist them in developing necessary policies and strategies to limit and control the COVID-19 pandemic [5].

The fast-paced growth of computer processing power allowed the utilization of Agent-Based Models (ABMs) to widely build simulation models for outbreaks [6]. Although experts are building many models to simulate and forecast the infection's trajectories, they do not cover the sophisticated behavioral and social aspects of societies that are exposed to this outbreak [7]. In this research, a survey was conducted on several published papers that used $\mathrm{ABM}$ to simulate infectious diseases outbreaks in different scenarios and environments. This review aims to understand the 
various uses and limitations of the ABS in building diseases outbreak models.

\section{Literature review}

\subsection{Modelling pandemics}

To better understand how a pandemic grows and how human intuition fails, it is first essential to understand the concept of exponential growth (1). It describes how quantities increase over time. In the early days of a pandemic, the growth rate looks like a linear growth, until the window of action had passed. This was particularly true with COVID-19 (Figure 1).

$$
X_{t}=X_{0}(1+R)^{t}
$$

Moreover, another feature of the growth of the positive cases was that the numbers were time-latent. This means that the positive cases are not identified until infected individuals had already been sick for several days and have likely infected other individuals [8]. Therefore, humanity realized the importance of finding new ways to help understand trajectories of pandemics and implement necessary policies to limit and prevent outbreaks such as COVID19.

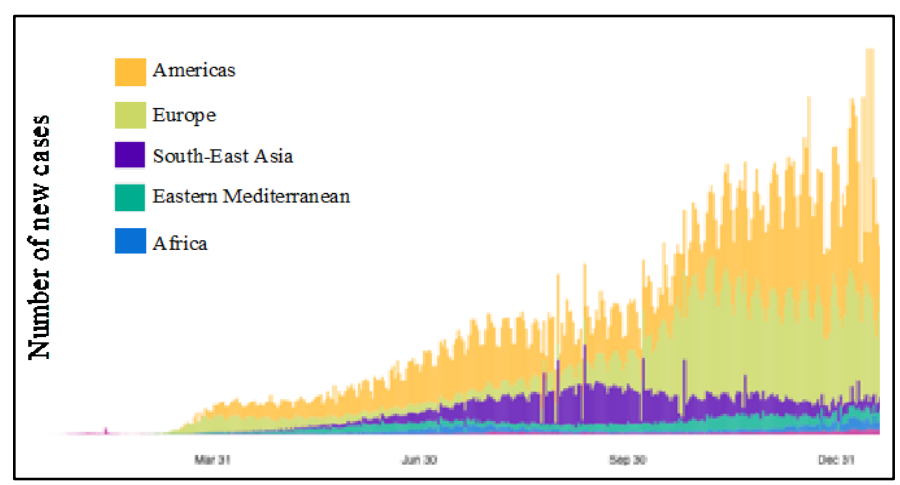

Figure 1: Growth of COVID-19 cases in the world in 2020 [1].

Modelling pandemics are rooted in traditions that go back to the 1920s, when mathematical equations were applied to simulate and model the distribution of the disease in populations, including positive cases, recovered cases and fatality rates. While these mathematical-derived approaches helped experts to understand the threshold, nature and herd immunity of plagues; it did not explain social and behavioral factors such as an individual's behavioral response towards public policies, measures, or the impact of mixed social contact on spreading trajectories [7].

\subsection{Agent-based Models (ABMs)}

In the early 1990s, the agent-based simulation was known as a third way of doing science. The field has substantially transformed since and found its way to the primary science field. Moreover, many ABS frameworks have been developed, such as RePast, AnyLogic and NetLogo. In addition, the open database of ABS models allowed swift and decisive prototyping of model developments [9]. For this paper, we can define the ABM as a computational simulation model, where agents are behaving independently without an external force. The agents' actions are usually a response to the situation they are exposed to during the process of the simulation [10]. Unlike other simulation models, ABMs are described as a simpler one. This type of models do not use a complicated set of rules or advanced architectures. Nevertheless, they can generate a variety of complicated patterns (behaviors) based on the modelling attributes caused by its simple agent interaction [11].

\subsection{Gaps in Agent-Based Models}

While mathematical concepts can be concisely defined and described, the same cannot be said of simulation models. Describing early ABMs was challenging to write or understand. No one knew where to locate the different types of information. As a result, the descriptions were often incomplete, and reproducing the model was ultimately impossible [12].

Although many signs of progress were being made in modelling pandemics through agent-based simulations, whenever a new pandemic occurs, similar modelling issues repeatedly arise and require precise attention. These issues include: (1) predicting sophisticated results when the vital data are not available nor reliable (2) reusing the model in a different setting that it was not designed for, and (3) not carefully maintaining good standards, practices or procedures, either for a race to academic recognition or for an immediate response to political pressure [7]. In contrast, [11] proposed a standard format for describing ABMs, known as the "Overview, Design, Details" concept (ODD). ODD was one of the initiatives to counter reoccurring challenges faced, in what has been known as "replication crisis". ODD was developed to make it easier to write, read, implement and replicate ABM. It can include mathematical and short algorithms and is structured into seven steps, as shown in Figure 2. These steps are grouped into three categories, Overview, Design and Details.

\begin{tabular}{|c|c|c|}
\hline & \\
\hline \multirow{5}{*}{$\mathbf{0}$} & 1 Purnoce and nattornc & Basic principles \\
\hline & Entitiec ctato & Emergence \\
\hline & 2. Entities, state variables and scales & Adaptation \\
\hline & $\begin{array}{l}\text { 3. Process overview and scheduling } \\
\text { Submodel } A\end{array}$ & Objectives \\
\hline & Submodel B & Learning \\
\hline D & 4. Design concepts & Prediction \\
\hline \multirow{5}{*}{ D } & 5. Initialization & Sensing \\
\hline & 6. Input data & Interaction \\
\hline & 7. Submodels & Stochasticity \\
\hline & Submodel A (Details) & Collectives \\
\hline & Submodel B (Details) ... & Observation \\
\hline
\end{tabular}

Figure 2: Overview, Design, Details (ODD) concept

\section{Methods}

\subsection{Methodological approach}

Document analysis is one of the effective and efficient ways to start the collection of data on a certain topic. Published articles can supply recent and broad data coverage [13]. Search Engines can facilitate faster data analysis. For this research, the critical survey approach was chosen to conduct a primary research, to understand the various uses and limitations of ABS, in building diseases outbreak models.

\subsection{Data Collection Plan}

Major databases were searched including ScienceDirect, Google Scholar and Crossref for (“Agent-based simulation”) OR 
(“Agent-based Model") OR ("Individual-Based Model") AND ("COVID-19"). Since the focus was COVID-19, and the pandemic only started spreading in early 2020 ; the time frame was set for all published journal articles in 2020 up to the $19^{\text {th }}$ of October 2020. The search was conducted without language restrictions; finally, all non-mathematical computing models articles were excluded.

\subsection{Quality of papers}

The focus subject and the time frame of the surveyed papers are relatively new; therefore, high citations was not a good indicator. Alternatively, Scimago Journal \& Country Rank ranking (SJR) of [14] was used. Most of the examined papers are ranked between Q1 \& Q3. Afterwards, if a paper was not published in a journal yet, the authors' credibility were examined via the ORCID database [15] to check if they have previously published in high ranked journals.

Table 1: Quality assessment checklist

\begin{tabular}{|l|l|}
\hline$\#$ & Question \\
\hline 1 & Is the paper contained in a high ranked journal? \\
\hline 2 & Is the paper cited by more than 5? \\
\hline 3 & $\begin{array}{l}\text { Have the Authors published before in a high-ranking } \\
\text { journal? }\end{array}$ \\
\hline 4 & $\begin{array}{l}\text { Is the paper providing clear purpose, method and } \\
\text { findings? }\end{array}$ \\
\hline
\end{tabular}

The papers' quality was evaluated against the quality assessment checklist (Table 1). Each answer has a value, and three different values determine the answer to each question. Questions answered with 'Yes' have been assigned with a value of (1), questions answered with 'No' have been assigned with a value of (0), while questions partly answered have been assigned with a value of (0.5). All papers that scored above $60 \%$ were included in the analysis (Table 2).

Table 2: Quality assessment result

\begin{tabular}{|l|l|l|l|l|l|l|}
\hline Source & Q1 & Q2 & Q3 & Q4 & Total & \% \\
\hline R01 & 1 & 1 & 1 & 1 & 4 & $100 \%$ \\
\hline R02 & 1 & 1 & 1 & 1 & 4 & $100 \%$ \\
\hline R03 & 1 & 1 & 1 & 1 & 4 & $100 \%$ \\
\hline R04 & 0 & 0.5 & 1 & 1 & 2.5 & $63 \%$ \\
\hline R05 & 0.5 & 0.5 & 1 & 1 & 3 & $75 \%$ \\
\hline R06 & 1 & 1 & 1 & 0.5 & 3.5 & $88 \%$ \\
\hline R07 & 1 & 1 & 1 & 1 & 4 & $100 \%$ \\
\hline R08 & 1 & 0 & 1 & 1 & 3 & $75 \%$ \\
\hline R09 & 1 & 1 & 1 & 1 & 4 & $100 \%$ \\
\hline R10 & 1 & 0.5 & 1 & 1 & 3.5 & $88 \%$ \\
\hline
\end{tabular}

\subsection{Selection of papers}

Ten journal articles that matched the research criteria were identified. Table 3 shows each study along with the journal's name, ranking, model used, and its availability. The following table (Table 3) shows the main qualified articles for the further analysis.
Table 3: Names of authors, source and journal and their SJR Quartiles rank

\begin{tabular}{|c|c|c|c|c|}
\hline $\begin{array}{l}\text { N } \\
\text { o. }\end{array}$ & $\begin{array}{l}\text { Journal } \\
\text { Name }\end{array}$ & $\begin{array}{l}\text { SJR } \\
\text { Quar } \\
\text { tiles }\end{array}$ & Model Availability & Country \\
\hline $\begin{array}{l}\mathrm{R} \\
01\end{array}$ & $\begin{array}{l}\text { Chaos, } \\
\text { Solitons } \\
\text { and } \\
\text { Fractals }\end{array}$ & Q1 & $\begin{array}{l}\text { https://bit.ly/COVID19_ABSs } \\
\text { ystem }\end{array}$ & Brazil \\
\hline $\begin{array}{l}\mathrm{R} \\
02\end{array}$ & $\begin{array}{l}\text { Nature } \\
\text { Human } \\
\text { Behavio } \\
\text { ur }\end{array}$ & Q1 & $\begin{array}{l}\text { Available from the } \\
\text { corresponding author upon } \\
\text { request. }\end{array}$ & USA \\
\hline $\begin{array}{l}\mathrm{R} \\
03\end{array}$ & $\begin{array}{l}\text { Chaos, } \\
\text { Solitons } \\
\text { and } \\
\text { Fractals }\end{array}$ & Q1 & $\begin{array}{l}\text { https://github.com/Institutefor } \\
\text { DiseaseModeling/covasim. } \\
\text { https://github.com/Jasminapg/ } \\
\text { Covid-19-Analysis. }\end{array}$ & UK \\
\hline $\begin{array}{l}\mathrm{R} \\
04 \\
\end{array}$ & $\begin{array}{l}\text { arXiv.o } \\
\text { rg }\end{array}$ & - & No & Germany \\
\hline $\begin{array}{l}\mathrm{R} \\
05\end{array}$ & $\begin{array}{l}\text { Informa } \\
\text { tics in } \\
\text { Medicin } \\
\text { e } \\
\text { Unlock } \\
\text { ed }\end{array}$ & Q3 & No & Iran \\
\hline $\begin{array}{l}\mathrm{R} \\
06 \\
\end{array}$ & JASSS & Q1 & No & Italy \\
\hline $\begin{array}{l}\mathrm{R} \\
07\end{array}$ & $\begin{array}{l}\text { Comput } \\
\text { ers in } \\
\text { Biology } \\
\text { and } \\
\text { Medicin } \\
\text { e }\end{array}$ & Q2 & Pseudo-code & Mexico \\
\hline $\begin{array}{l}\mathrm{R} \\
08 \\
\end{array}$ & $\begin{array}{l}\text { Safety } \\
\text { Science }\end{array}$ & Q1 & No & Chile \\
\hline $\begin{array}{l}\mathrm{R} \\
09\end{array}$ & $\begin{array}{l}\text { Mathem } \\
\text { atical } \\
\text { Bioscie } \\
\text { nces }\end{array}$ & Q2 & $\begin{array}{l}\text { https://www.github.com/gress } \\
\text { man/covid_university }\end{array}$ & USA \\
\hline $\begin{array}{l}\mathrm{R} \\
10\end{array}$ & $\begin{array}{l}\text { Buildin } \\
\text { g and } \\
\text { Environ } \\
\text { ment }\end{array}$ & Q1 & No & China \\
\hline
\end{tabular}

\section{Results}

After analyzing the main contribution of each article, the following are the main identified themes of the studies.

\subsection{Agent-Based model of health and economic effect simulation}

The authors of [2] pointed out that it is a challenge for decision and policy makers with only limited studies available on the pandemic.. This paper proposed an ABM simulating the viral and economic impact of COVID-19 in a closed environment. The results can be widely used by decision and policy makers to evaluate the effectiveness of different social policies in a real-life scenario.

The proposed ABM is simulating a closed society living in a shared limited environment. The community consists of individuals and groups of families, businesses and a government. Each agent is interacting with each other. The given characterization by the model is supposed to emulate the character of a community. 


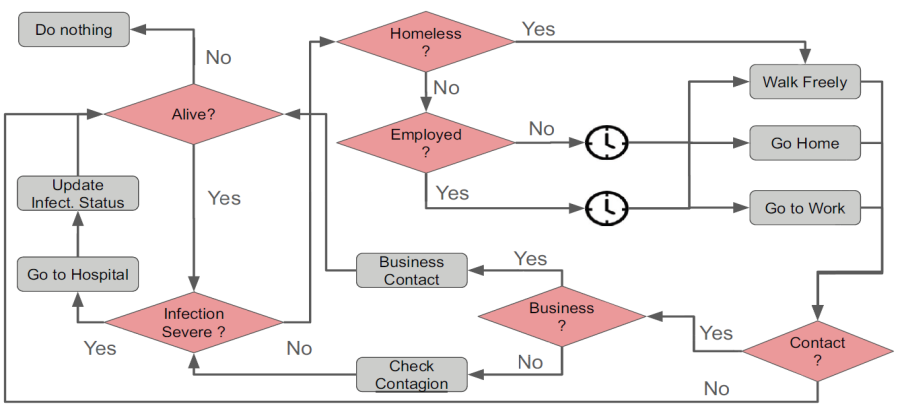

Figure 3: Agent activity cycle by [2].

The paper of [2] studied seven different scenarios using variable social inputs. The findings revealed that a lockdown or a semi-lockdown was the best-forecasted scenario to save lives, slow down viral spreading rates and lessen infection and fatality rates. Moreover, since the complete isolation was not practical, a 50 per cent lockdown scenario with social distance and mask usage was the best policy to save lives. On the other hand, the vertical isolation was ineffective (only persons below 18 years of age and seniors stay at home) was like a 'doing nothing' scenario. These results proved that COVID-19-ABS modelling was able to effectively generate social scenarios, matching several similar studies [2].

Although applying ultimate measures against the pandemic is successfully limiting fatality rates and hospitalizations, it does not mean societies are saved. Without herd immunity as well, a second wave is likely to take place when restrictions are lifted, and rules on taking protective measures are relaxed. In these scenarios, it is vital to analysis different containment strategies to limit the probabilities of other waves, and at the same time, balancing health and economic objectives.

\subsection{Agent-based simulation using mobility data with demographic data}

In another study [3] focus was made on preventing second waves of the pandemic, by simulating the impact of testing, contact tracing and staying at home. The research used an estimation of population communication patterns between residences to create a detailed ABM. The research used detailed data on transportation, social activities and demographics, and generated a network that coded the patterns of contact for 85,000 agents in an area, for six months. The agents were used to simulate different census locations in the Boston area in the United States. This research followed the same methodology used by [4] in their paper "Measurability of the epidemic reproduction number in datadriven contact networks data-driven contact networks".

The generated network was defined by a proposed multilayer network, forming the basis of social interventions on three layers, namely the school layer, workplace and community layer and house household layer, as displayed in Figure 3.

The estimated intervention between the two agents in the workplace and community layer is calculated from the data by calculating the probability of a mutual meeting between two individuals in a particular place (for example, at a food court or a shop) and using the time spent in the same place as the contributing factor.

www.astesj.com

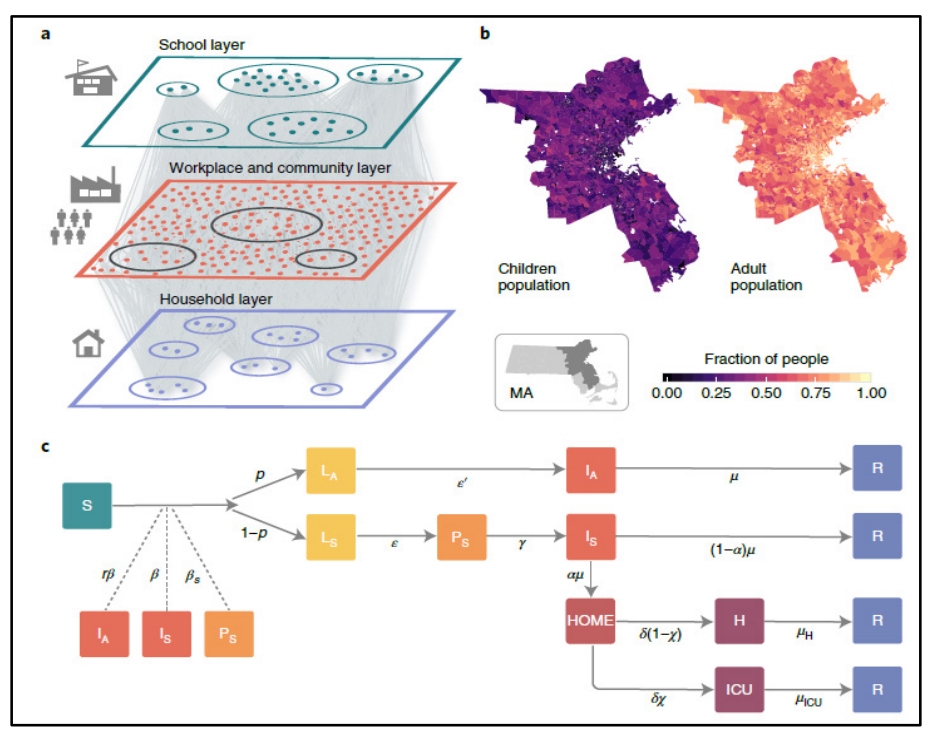

Figure 4: Model component as described by [3] "Susceptible (S), latent asymptomatic (LA), latent symptomatic (LS), presymptomatic (PS), infectious asymptomatic (IA), infectious symptomatic (IS), hospitalized (H), hospitalized in intensive care (ICU) and recovered (R) individuals" [4].

In [4], the author found that the best intervention policies to limit and control a second wave is by imposing a period of strict social distancing, followed by a healthy level of testing. In addition, an effective way of undergoing contact-tracing and home quarantine can keep the pandemic within the limit of the public health care systems in Boston, with the possibility of opening up the economy. While the herd immunity is absent, the finding states that a response system based on testing enforcements and contacttracing can have a significant role in limiting and relaxing the restriction rules.

\subsection{Agent-based simulation in the educational context}

Just like the previous papers, [16] aimed their efforts to limit the impact of the second COVID-19 epidemic wave, but within the education context, specifically in UK schools. The research used an ABM called "Covasim". This model was developed in the United States and calibrated to the UK's outbreak parameters. This paper seems to agree with [3]'s findings on the role of large-scale testing, and effective contact-tracing, followed by effective isolation of infected individuals.

However, the research of [17], which also conducted their simulation in the education sector, had additional useful insights. Although both previous papers by [16] and [3] concluded the importance of wide-scale testing, they might have neglected another critical factor. The authors of [17] had pointed out the issue of testing accuracy, and how it is a critical factor that needs to be swiftly addressed. Moreover, the authors also discussed three significant and expected findings that will impact the education sector: (1) Large classes will be significantly risky with increasing infection rates, (2) students should strictly hold-off all sorts of contact with other individuals, and (3) teachers need to be ready for extended students' absence mainly because of being held in quarantine.

\subsection{Agent-Based simulation built on pedestrian dynamics}


The authors of [6] are suggesting another Agent-Based simulation based on pedestrian dynamics. This paper analyzed how pedestrians behave in open areas in conditions with viral spreading, such as with COVID-19. This evaluation was done to gather useful insights about exposure time, and the effectiveness of social distancing measures, as stated by the German government (1:5m), at "an infection rate of $2 \%$ ".

The paper provided a different scenario of pedestrian dynamics in realistic situations while focusing on contagious diseases. The simulation was based on ABM works including "A Mathematical model for the behavior of pedestrians" (1991), "Simulating dynamical features of escape panic" (200), "Self-organizing pedestrian movement" (2001) and "Simulation of pedestrian crowds in normal and evacuation situations" (2002).

The simulation conducted a pedestrian scenario in an ideally sized German supermarket covering 4,800 square meters $(80$ × 60 meters), including shelves and counters as shown in Figure 5. There were 34 destinations identified as "point of interest" with a set of agents being defined as "infected".

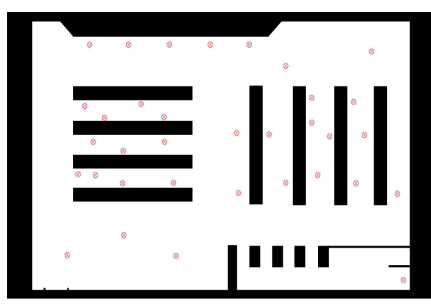

(a) Scene with destinations shown as red circles with crosses inside

Figure 5: Supermarket layout with walking spaces in white and obstacles in black

The experiment conducted using ABS revealed promising and valuable results for policy makers. The experiment assumed that if the infection rate was $2 \%$, a minimum distance of $1: 5 \mathrm{~m}$ can be kept if the density is not exceeding one individual for every 16 square meters. The proposed model can be a foundation to better assess the risks in public places within the circumstances of contagious diseases [6].

\subsection{Spatio-temporal Agent-Based simulation}

The study of [5] investigated the impact of different closure scenarios to control and limit the COVID-19's spread, including schools, educational institutes and the workplace, in addition to social distancing practices in the city of Urmia, Iran. In this paper, the COVID-19 outbreak was simulated with an ABM using a described set of behaviors in a defined environment. The SEIRD model (Susceptible Infectious Recovered Deceased Model) was used to mimic the infection of a human agent. Then, an allcontrolling plan was applied to the model.

The attribute of the ABM's environment cells was defined based on the spatial data. The related value was defined based on the spital data layers, as shown in Figure 6.

The simulation results indicated that closure of schools and educational institutes (for 50 days between the $21^{\text {st }}$ of February the $10^{\text {th }}$ of May 2020) decreased the infection rate by an average of 4.94 per cent weekly and a total of 49 per cent. While working from home at $30 \& 70$ per cent of Urmia city's population (from the $21^{\text {st }}$ of February 2020 - the $10^{\text {th }}$ of May 2020) decreased the infection rate by $3.30 \& 5.25$ per cent weekly and $32.98 \& 52.48$ per cent in total. Consequently, applying social distancing (from the $27^{\text {th }}$ of March 2020 at $30 \& 70$ per cent of Urmia city's population), led to a decrease in the infection rate at $5.24 \& 10.07$ per cent weekly, in addition to $31.46 \& 60.44$ per cent in total. The main finding recommended applying social distancing to the majority of the population. The paper claimed their novelty was in the model itself, but the model's source code was not provided or shared.

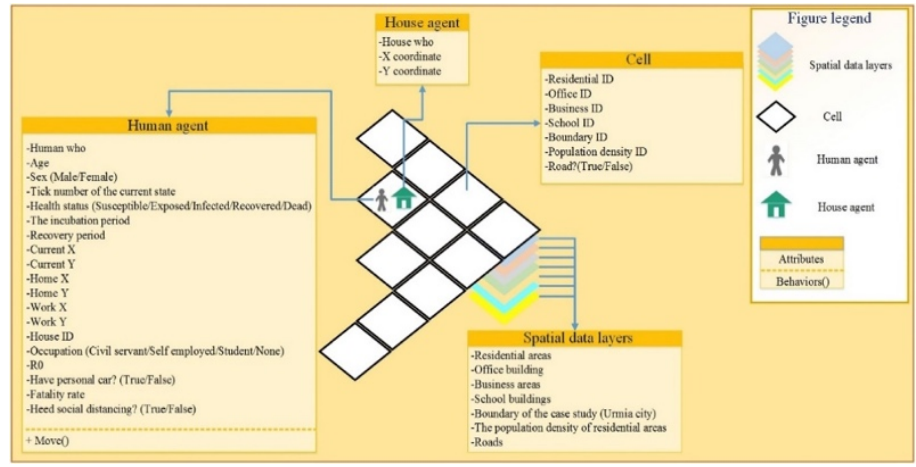

Figure 6: The elements of the Spatio-temporal ABM and their details

\subsection{Simulating COVID-19 in facilities}

The paper of [11] presented an $\mathrm{ABM}$ to evaluate the transmission risks in building environments. The paper tested several scenarios to evaluate and validate the proposed model. The model has been built as an iterative scheme consisting of a Pseudocode. It was adopted and modified from the work of Professor Hiroki Sayama.

The paper provided the code lines which was considered as simple and can be coded efficiently. According to the study, the model showed useful and promising results that may help decisionmakers to determine optimal strategies to reduce infection rates inside facilities and closed environments. The entire process of the model's operation is summarized in Figure 7.

\subsection{Simulation of COVID-19 in the construction sector's context}

The author of [18] examined another vital sector that little is known about. The study focused on the outbreak of COVID-19 among construction workers using an ABM. The paper's aim was no different from that of other papers, which was examining different intervention strategies. However, there was probable novelty in examining the pandemic's spread in the context of the construction environment. The approach used was classifying the risk of activities for the workers between low, medium and high, followed by simulation of the spread in a construction project. The result of the simulation recommended a reduction in workforce in construction projects between 30 to 90 per cent. However, it wasmentioned that a limitation of the analysis might result in the oversimplification of the realistic condition. Nevertheless, the finding of the study can be used as a basis to simulate the potential of second waves after relaxing the social distancing restrictions in regions that have heavy construction works. 


\begin{tabular}{|c|c|c|}
\hline \multicolumn{3}{|c|}{ Algorithm 1. Pseudo-code for the COVID-19 transmission risk model } \\
\hline \multirow{2}{*}{$\begin{array}{l}1 . \\
2 .\end{array}$} & \multicolumn{2}{|c|}{ Input: $A, B, l_{i n}, u_{i n}, l_{c m}, u_{c m},\left[L_{x}, U_{x}, L_{y}, U_{y}\right], \alpha$, maxiter $, R, S, k=1$} \\
\hline & $\operatorname{Pri}_{i} \leftarrow$ InitializeProbInf $\left(A, l_{i n}, u_{i n}\right)$ & \multirow{3}{*}{ Initialization } \\
\hline 3. & $\operatorname{Prcm}_{i}^{\mathbf{A}}, \operatorname{Prcm}_{j}^{\mathbf{B}} \leftarrow$ InitializeProbMob $\left(A, B, l_{c m}, u_{c m}\right)$; & \\
\hline 4. & $\mathbf{A}(k), \mathbf{B}(k) \leftarrow$ InitializePos $\left(A, B,\left[L_{x}, U_{x}, L_{y}, U_{y}\right]\right)$ & \\
\hline 5. & \multicolumn{2}{|l|}{ while $k<=$ maxiter do } \\
\hline 6. & For each $\mathbf{a}_{i}(k) \in \mathbf{A}(k)$ & \multirow{9}{*}{ Rule I } \\
\hline & $F \leftarrow$ FindAnInfectedAgentInNeighbor $(\mathbf{B}(k), R)$ & \\
\hline & If $(F==1)$ then & \\
\hline & If $\left(\right.$ rand $\left.\leq P r i_{i}\right)$ then & \\
\hline & $\mathbf{A}(k) \leftarrow$ DeleteFromA $\left(\mathbf{a}_{i}\right)$ & \\
\hline 11. & $\mathbf{B}(k) \leftarrow \operatorname{Includeln} \mathbf{B}\left(\mathbf{a}_{i}\right)$ & \\
\hline & end If & \\
\hline 13. & end If & \\
\hline 14. & end for & \\
\hline & for each $\mathbf{a}_{i}(k) \in \mathbf{A}(k)$ & \multirow{11}{*}{ Rule II for $\mathbf{A}$} \\
\hline & If $\left(\right.$ rand $\left.\leq \operatorname{Prcm}_{i}^{\mathrm{A}}\right)$ then & \\
\hline & If $($ rand $\leq \alpha)$ & \\
\hline & $\mathbf{a}_{i}(k+1) \leftarrow$ LocalMovement $\left(\mathbf{a}_{i}(k), S\right)$ & \\
\hline & else & \\
\hline & $\mathbf{a}_{i}(k+1) \leftarrow$ LongMovement $\left(\left[L_{x}, U_{x}, L_{y}, U_{y}\right]\right)$ & \\
\hline & end If & \\
\hline 22. & else & \\
\hline & $\mathbf{a}_{i}(k+1)=\mathbf{a}_{i}(k)$ & \\
\hline 24. & enf If & \\
\hline 25. & end for & \\
\hline & for each $\mathbf{b}_{j}(k) \in \mathbf{B}(k)$ & \multirow{11}{*}{ Rule II for B } \\
\hline & If $\left(\right.$ rand $\left.\leq \operatorname{Prcm}_{j}^{\mathrm{B}}\right)$ then & \\
\hline & If $($ rand $\leq \alpha)$ & \\
\hline & $\mathbf{b}_{i}(k+1) \leftarrow$ LocalMovement $\left(\mathbf{b}_{i}(k), S\right)$ & \\
\hline & else & \\
\hline & $\mathbf{b}_{i}(k+1) \leftarrow$ LongMovement $\left(\left[L_{x}, U_{x}, L_{y}, U_{y}\right]\right)$ & \\
\hline & end If & \\
\hline & else & \\
\hline & $\mathbf{b}_{i}(k+1)=\mathbf{b}_{i}(k)$ & \\
\hline & enf If & \\
\hline 36. & end for & \\
\hline & $\begin{array}{l}k=k+1 \\
\text { end while }\end{array}$ & \\
\hline
\end{tabular}

Figure 7: Proposed ABS model algorithm by [11]

\subsection{Simulation accuracy in a responsible manner}

Within the context of numerous simulations available and under the pressure of polices makers, [7] presented a paper proposing guidelines, urging the scientific community to follow, so they can handle the transparency and speediness of their findings in a responsible manner.

While many experts have provided sound simulations and prediction of the COVID-19 trajectories; their papers might not cover the complete sophisticated behavioral and social parameters.

Therefore, [7] called for urgent steps to improve the quality of ABMs. The first proposed step was to examine previous ABMs of COVID-19 and explore their potentials, identify gaps, and suggest counter measures. The counter measures would aid in the rapid demand of immediate responses, which is a critical step to avoid collective mistakes. The second suggestion was to consider the pressure between modelling efforts and policy makers, and develop a better understanding of the challenges which aroused from the over expectation of the emerged knowledge, due to misinterpreting the science of modelling. Finally, measures to address these gaps and improve the relationship between the sciences and public policies was suggested. These included a call for a wide-scale cooperation between the public and academic stakeholders, where knowledge, models and data can be exchanged and shared.

The call to action by [7] can be summarized into two major actions. First was a call to modelers to maintain high standards when building ABMs. The second was for institutional agencies who own useful data that can help calibrate and inform COVID19 models, to engage with trusted academic associations to make this data available for the rest of the scholars.
Moreover, the paper recommended to follow three best practices that are already well known among agent-based modelers: (1) building models based on open sources such as MASON and NETLOG, (2) following standing documenting protocols, (3) using long-lasting online content storage tools such as ComSES, to ensure the model is fully documented prior to submission. The paper urged scholar modelers to follow these guidelines, which are very critical during this exceptional times, to enhance their credibility.

\subsection{Summary}

The following Table 4, summarizes all the examined papers, including article code $(\mathrm{N})$, the main purpose, method used with a model screenshot, the main findings and country (C) of the experiment. The summary excludes the results of [7]. As it is proposing ABM simulation documentation guidelines for the scientific community to follow. However, it has been included for its high importance and relevance to the overall survey.

Table 4: Summary of the analyzed papers

\begin{tabular}{|c|c|c|c|c|}
\hline $\mathrm{N}$ & Purpose & Method / Model screenshot & Main Finding & $\mathrm{C}$ \\
\hline$\widetilde{\sigma}$ & $\begin{array}{l}\text { To simulate } \\
\text { health and } \\
\text { economic } \\
\text { effects of } \\
\text { social } \\
\text { distancing } \\
\text { interventions }\end{array}$ & $\begin{array}{l}\text { Figure 8: COVID-ABS: a } \\
\text { new SEIR ABM }\end{array}$ & $\begin{array}{l}\text { "A useful tool to } \\
\text { assist politicians } \\
\text { and health } \\
\text { authorities to plan } \\
\text { their actions against } \\
\text { the COVID-19" } \\
\text { [2]. }\end{array}$ & 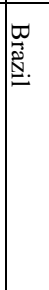 \\
\hline గ్ర & $\begin{array}{l}\text { To simulate } \\
\text { the impact of } \\
\text { testing, contact } \\
\text { tracing } \\
\text { and household } \\
\text { quarantine on } \\
\text { second waves } \\
\text { of } \\
\text { COVID-19 }\end{array}$ & 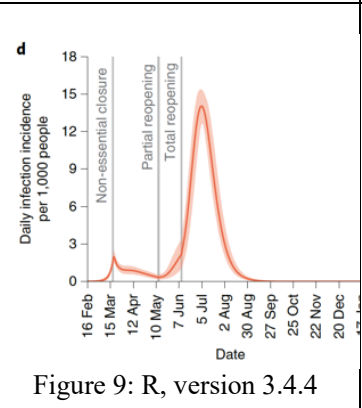 & $\begin{array}{l}\text { "A response system } \\
\text { based on enhanced } \\
\text { testing } \\
\text { and contact tracing } \\
\text { can have a major } \\
\text { role in relaxing } \\
\text { social-distancing } \\
\text { interventions in the } \\
\text { absence of herd } \\
\text { immunity against } \\
\text { Covid-19" [3]. }\end{array}$ & $\begin{array}{l}\sigma \\
\sim \\
D\end{array}$ \\
\hline ర్లి & $\begin{array}{l}\text { To Determine } \\
\text { the optimal } \\
\text { strategy for } \\
\text { reopening } \\
\text { schools }\end{array}$ & 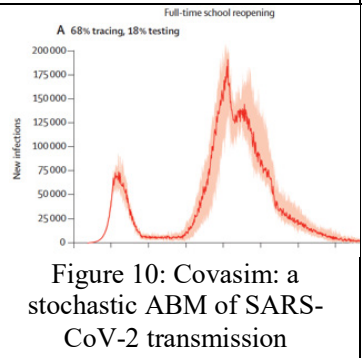 & $\begin{array}{l}\text { "A comprehensive } \\
\text { and effective test-- } \\
\text { trace-isolate } \\
\text { strategy would be } \\
\text { required to avoid a } \\
\text { second COVID-19 } \\
\text { wave" [16]. }\end{array}$ & $\underset{\pi}{\overparen{\pi}}$ \\
\hline 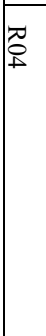 & $\begin{array}{l}\text { To simulate } \\
\text { Pedestrian } \\
\text { dynamics for } \\
\text { exposure time } \\
\text { estimation }\end{array}$ & $\begin{array}{l}\text { Figure 11: ABM based on the } \\
\text { model of Helbing et al. }\end{array}$ & $\begin{array}{l}\text { "Results suggest } \\
\text { that a density of } \\
\text { one person per } \\
16 \mathrm{~m}^{2} \text { or below is } \\
\text { sufficient" [6]. }\end{array}$ & 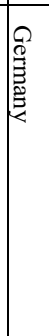 \\
\hline
\end{tabular}




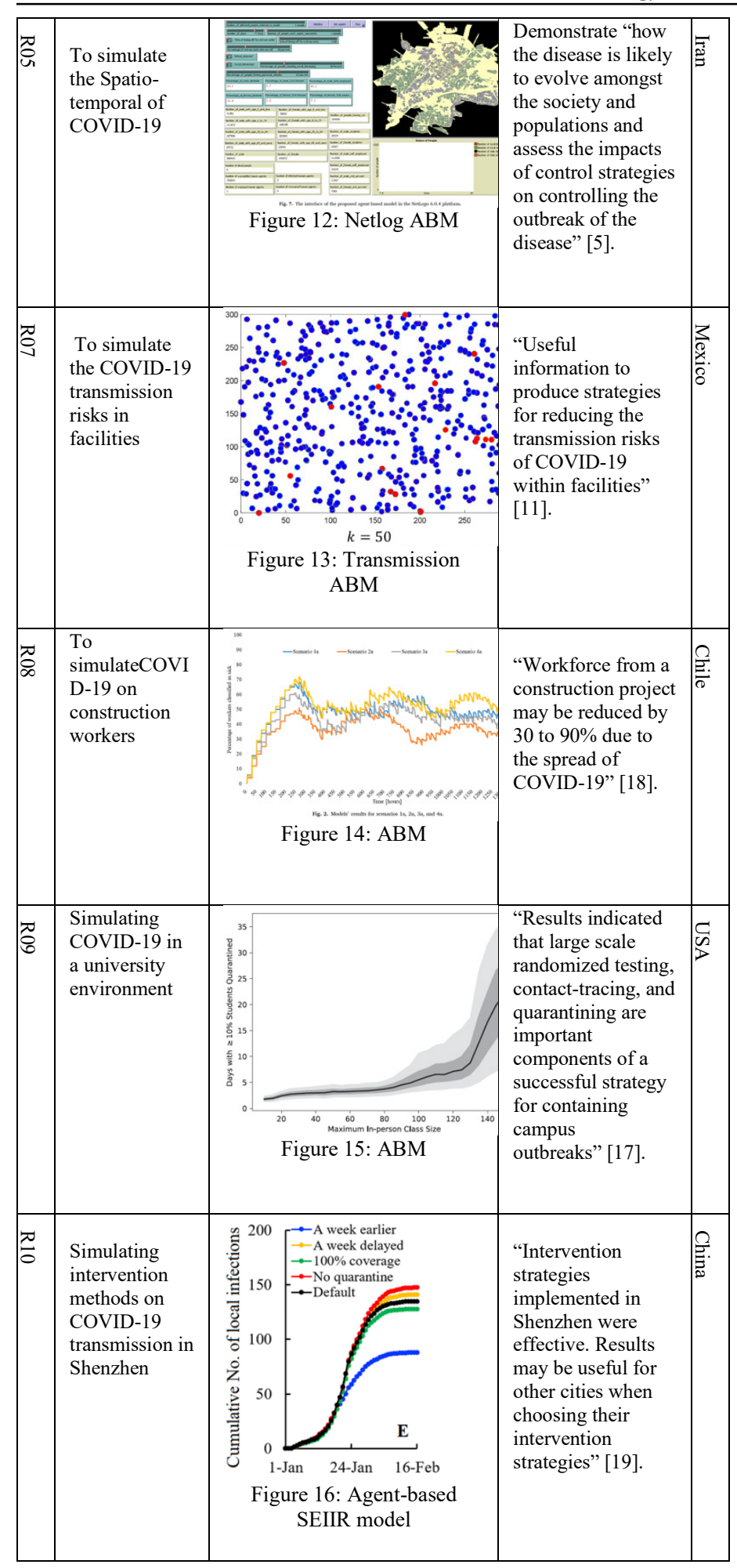

\section{Discussion}

This paper examined ten research reports adapting ABMs to simulate the impact of COVID-19. Paper [11] presented an ABM to assess the transmission risk in building environments. However, this model was not the only one. [2] also proposed a new Agentbased simulation with promising results. The presented ABS model was not only simulating the transmission risks, but it also emulated the economic impact of businesses and the government. At the same time, an SEIR ABM simulating pandemic outbreaks with the help of a society of agents called COVID-ABS was presented.

Furthermore, other researchers tried to combine more than one tool. In [5], the author used Spatio-temporal simulation to present the impact of various pandemic preventing strategies, including closures, social distancing, working and studying from home in Urmia, Iran. Other researchers also examined these strategies in Boston, United States. In [3], the author combined more than one instrument. In order to build a detailed ABS simulating a second wave; anonymized, geolocalized mobility data were integrated with census and demographic data. On the other hand, In [6] the author tried to approach pandemic controlling strategies from a different perspective. They focused on examining data on pedestrians behavior and collective time of exposure to evaluate the efficiency of social distancing measures within COVID-19's context.

The aims of the remaining researches were not in developing the model itself, but to use existing ones to support decisionmakers in identifying the right intervention strategies. Their novelty was the context of the simulation. In [19], the author presented and analyzed different preventing strategy in Shenzhen. In comparison, [16] conducted a simulation to determine an optimal strategy in the education sector. Likewise, [17] also examined different strategies and measures in the education sector, but their focus was university environments. In [18], the author has moved to a different sector, which is the construction sector. In [18], the author attempted to study the pandemic's impact on construction workers and the best way to lower the infection rate. Alternatively, [7] presented a paper proposing guidelines to ensure transparency and speediness of modelers' findings being made available, are done responsibly.

Most papers used ABMs in their methods except [7], which method and approach were not clear or defined. Generally, the findings of the proposed models were promising and have some useful insights.

The authors of [5] suggest future research to be conducted in a different region that has not been evaluated yet, using different parameters and attributes.

Among the surveyed papers, two significant findings related to widely shared believes were proven wrong: The paper of [19] found out that airport screening was not very useful. Forty-six per cent of infected cases were not detected during airport screenings due to several reasons. Part of the failure was due to (1) the sensitivity of the entry and exit screening, (2) asymptomatic cases, and (3) the incubation period, while [2] found that vertical isolation policies promoted by some countries like Brazil and United States was similar to 'doing nothing' scenarios. In contrast, [19] reported that working and studying from home and mask-wearing were found to be the most effective policies. [2] seem to reach the same conclusions but in a more realistic manner. Their finding focused on combining wearing of masks and 50 per cent lockdown with social distancing. However, [16] and [3] have added wide-scaled testing as an additional layer of intervention policy. Although nation-wide testing might be theoretically useful, it does not take into consideration the social and behavioral aspect of individuals in addition to their economic situation, where testing is not free in some regions. In addition, there are the issues of testing accuracy, 
as pointed out by [17] and the detectability rate as mentioned by [19].

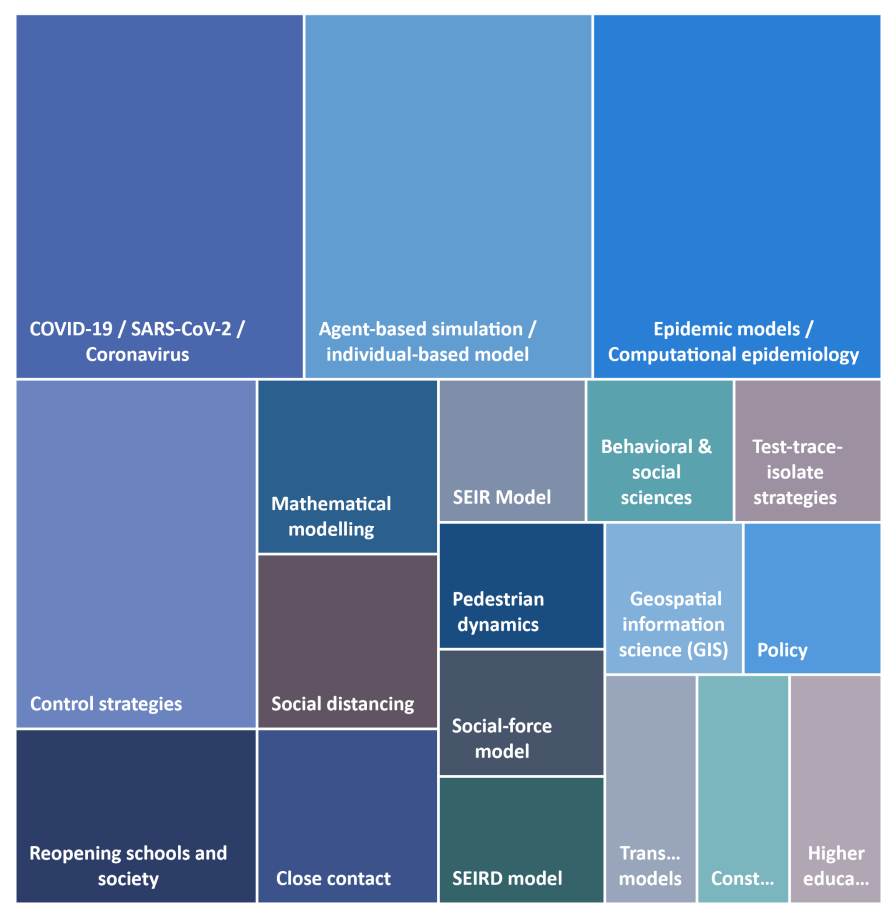

Figure 17: Most discussed subjects based on keywords

\section{Limitation and Future work}

Many noteworthy articles where missed due to the limited knowledge of different keywords and synonyms used by scholars. Figure 17 shows different keywords commonly used by the examined papers. Moreover, many scholars use arXiv (arXiv.org) e-Print archives as a swift free distribution service with open access. However, papers published in these databases are by invitations only, and not peer reviewed. Therefore, it is not recognized by Scimago's Journal \& Country Rank ranking.

\section{Conclusion}

This paper examined the different discussions on the COVID19 pandemic's simulations that used ABMs. It was found that agent-based simulation for COVID-19 was used in a variety of contexts, including schools, universities, workplaces, facilities and construction. Methods used varied from simple ones consisting of a few lines of code, to sophisticated models combining mobility data with demographic data or Spatio-temporal data, or from different perspectives such as ABS based on pedestrian dynamics. The main findings of this analysis was in line with works of literature, where mask-wearing and social distancing are among the best strategies, and against some common believes, airport screening and vertical isolation were less effective. The main limitation and future suggestions are on improving the quality of papers by following modelling guidelines proposed by ODD, validating the test accuracy, and considering the behavioral and social attributes of individuals in different cultures and regions.

Outbreaks are usually very sophisticated phenomena, and no one specific model can be applied to all cities of the world [5]. The different parameters do not only impact the infection rates, but they also vary from one place to another. These parameters include but are not limited to culture differences, literacy, awareness, interactions, transportation, the urban context, population density, job diversity, age variations, gender, number of employees, number of students and people with private transportation means. These only few of the parameters that impact the way the outbreak spreads in different regions. The papers recommended that similar simulations be conducted in a different part of the world using different parameters and different controlling and preventing strategies.

\section{Acknowledgment}

This study would not be possible without the guidance of Professor Dr Piyush Maheshwari. The study was a part of a project done at the British University in Dubai. The authors declare no conflicts of interest.

\section{References}

[1] WHO, Archived: WHO Timeline - COVID-19, World Health Organizaiton, 2020.

[2] P.C.L. Silva, P.V.C. Batista, H.S. Lima, M.A. Alves, F.G. Guimarães, R.C.P Silva, "COVID-ABS: An agent-based model of COVID-19 epidemic to simulate health and economic effects of social distancing interventions," Chaos, Solitons and Fractals, 139, 110088, 2020, doi:10.1016/j.chaos.2020.110088.

[3] A. Aleta, D. Martín-Corral, A. Pastore y Piontti, M. Ajelli, M. Litvinova, M. Chinazzi, N.E. Dean, M.E. Halloran, I.M. Longini, S. Merler, A. Pentland, A. Vespignani, E. Moro, Y. Moreno, "Modelling the impact of testing, contact tracing and household quarantine on second waves of COVID-19," Nature Human Behaviour, 4(9), 964-971, 2020, doi:10.1038/s41562-0200931-9.

[4] Q.-H. Liu, M. Ajellic, A. Aleta, S. Merler, Y. Moreno, A. Vespi, "Measurability of the epidemic reproduction number in data-driven contact networks," Proceedings of the National Academy of Sciences, 115(50), 12680-12685, 2018, doi:10.1073/pnas.1811115115.

[5] N. Mahdizadeh Gharakhanlou, N. Hooshangi, "Spatio-temporal simulation of the novel coronavirus (COVID-19) outbreak using the agent-based modeling approach (case study: Urmia, Iran)," Informatics in Medicine Unlocked, 20, 1-12, 2020, doi:10.1016/j.imu.2020.100403.

[6] T. Harweg, D. Bachmann, F. Weichert, AGENT-BASED SIMULATION OF PEDESTRIAN DYNAMICS FOR EXPOSURE TIME ESTIMATION IN EPIDEMIC RISK ASSESSMENT, 2020.

[7] F. Squazzoni, J.G. Polhill, B. Edmonds, P. Ahrweiler, P. Antosz, G. Scholz, E. Chappin, M. Borit, H. Verhagen, F. Giardini, N. Gilbert, "Computational models that matter during a global pandemic outbreak: A call to action," JASSS, 23(2), 1-14, 2020, doi:10.18564/jasss.4298.

[8] J. Liebowitz, The Business of Pandemics: The COVID-19 Story, CRC Press, 2020.

[9] J. Thaler, P.O. Siebers, "Specification testing of agent-based simulation using property-based testing," Autonomous Agents and Multi-Agent Systems, 34(2), 47, 2020, doi:10.1007/s10458-020-09473-8

[10] C. Macal, M. North, "INTRODUCTORY TUTORIAL: AGENT-BASED MODELING AND SIMULATION," in Proceedings of the 2014 Winter Simulation Conference, IEEE Press, 2014.

[11] E. Cuevas, "An agent-based model to evaluate the COVID-19 transmission risks in facilities," Computers in Biology and Medicine, 121, 1-12, 2020, doi:10.1016/j.compbiomed.2020.103827.

[12] V. Grimm, S.F. Railsback, C.E. Vincenot, U. Berger, C. Gallagher, D.L. DeAngelis, B. Edmonds, J. Ge, J. Giske, J. Groeneveld, A.S. A Johnston, A. Milles, J. Nabe-Nielsen, J. Gareth Polhill, V. Radchuk, M.-S. Rohwäder, R.A. Stillman, J.C. Thiele, D. Ayllón, K. ooo-----, "The ODD Protocol for Describing Agent-Based and Other Simulation Models: A Second Update to Improve Clarity, Replication, and Structural Realism," JASSS, 23(2), 2-20, 2020.

[13] G.A. Bowen, "Document analysis as a qualitative research method," Qualitative Research Journal, 9(2), 27-40, 2009, doi:10.3316/QRJ0902027.

[14] T. Krajna, SCImago Journal \& Country Rank, Polimeri (Zagreb), 29(4), 258-262, 2008.

[15] ORCID, 2020. 
[16] J. Panovska-Griffiths, C.C. Kerr, R.M. Stuart, D. Mistry, D.J. Klein, R.M. Viner, C. Bonell, "Determining the optimal strategy for reopening schools, the impact of test and trace interventions, and the risk of occurrence of a second COVID-19 epidemic wave in the UK: a modelling study," The Lancet Child and Adolescent Health, 4(11), 817-827, 2020, doi:10.1016/S2352-4642(20)30250-9.

[17] P.T. Gressman, J.R. Peck, "Simulating COVID-19 in a university environment," Mathematical Biosciences, 328(108436), 2020, doi:10.1016/j.mbs.2020.108436.

[18] F. Araya, "Modeling the spread of COVID-19 on construction workers: An agent-based approach," Safety Science, 133(105022), 2020, doi:10.1016/j.ssci.2020.105022.

[19] N. Zhang, P. Cheng, W. Jia, C.H. Dung, L. Liu, W. Chen, H. Lei, C. Kan, X. Han, B. Su, S. Xiao, H. Qian, B. Lin, Y. Li, "Impact of intervention methods on COVID-19 transmission in Shenzhen," Building and Environment, 180(107106), 2020, doi:10.1016/j.buildenv.2020.107106. 